\title{
Avebury Portal - A Location-Based Augmented Reality Treasure Hunt for Archaeological Sites
}

\author{
Farbod Shakouri, Feng Tian \\ Faculty of Science \& Technology, Bournemouth University, Bournemouth, \\ BH12 5BB, UK
}

\begin{abstract}
Many archaeological sites are less popular by visits amongst the younger group and overall less popular than majority of other heritage sites. They are often not enhanced by supporting medium like in museums or historic buildings. Many augmented reality (AR) systems have been developed for archaeological sites and proved to benefit user engagement. However, most result in superimposing a virtual reconstruction of the site for users to passively observe and lack exploration of other methods for designing an interactive engaging experience. In this paper, we demonstrate the development of a location-based treasure hunt AR app, Avebury Portal, for the heritage site; Avebury in England. Avebury Portal uses puzzles with the environment to give clues, and a narrative that responds to the user's location. We developed Avebury Portal with Unity Engine and Vuforia to demonstrate the effectiveness of using AR to enhance visitors' experiences on learning.
\end{abstract}

Keywords: Location-based AR, Archaeological Heritage Site, Interactive Learning, Multimodal Interface, Locative Narrative

\section{Introduction}

In 2017, UK Department for Digital, Culture Media \& Sport reported that visits to heritage sites of archaeological interest were lower amongst the younger group of 1624 , and overall lower than other heritage sites [1]. A contributing factor may be the lack of exciting mediums used on-site to exhibit the information about archaeological sites when compared to traditional museums, where information is displayed in a controlled environment and enhanced by supporting materials such as signs, photos, videos and even sound systems [2]. One technology recently has shown potential capabilities which help create such environment for outdoor archaeological sites, known as augmented reality (AR).

Augmented reality has become more accessible on mainstream devices like mobile smartphones as they are now capable of providing valuable information from various modes of data like geo-location, touch, etc. People amongst the same age group have shown a rising interest for AR apps like Pokémon Go [3], resulting in researchers finding ways to adopt this technology for productive implications. It is suggested that AR should be adopted in the next few years to provide new and creative ways for teaching, learning and research, according to 55 studies published between 2011 and 2016 [4]. 
Many augmented reality systems have been developed and deployed for cultural and archaeological sites where the system had contextual-awareness [5]. However, the focus of these applications was to reconstruct the heritage sites where the systems would visualise 3D models superimposed on their correspondent real-world structures. Ultimately, many of these previous applications did not integrate core framing context like liminal interface or Location Aware Narrative (LAN) to engage the users in a treasure hunt-like experience.

In this paper we propose an augmented reality application for outdoor, locationbased treasure hunt called Avebury Portal. Avebury Portal is a mysterious treasure hunt intended to enhance users' engagement with Avebury Henge. The Neolithic stone circle is located in Wiltshire, south west England and serves great importance to contemporary pagans. It was built and altered over many centuries from about $2850 \mathrm{BC}$ until about $2200 \mathrm{BC}$ [6] and is now a part of a village always open to the public for visits. Avebury Portal provides information using contextualised learning which enables users to explore and discover ancient artefacts at the heritage site by walking around with a mobile device to find virtual 'treasures' hidden on-site. The system uses a location-aware narrative system to give clues and generate puzzles, and guiding users with the provided 'smart map' that locates points of interest.

\section{Related Works}

AR applications have been researched and developed for historical and cultural environments. It is often extended to a complete methodology for real-time mixed reality system that features a simulated animation scene, re-enacted by virtual characters [7]. The visitors can observe the animated characters perform in a storytelling drama on the site using mobile mixed reality (MR) glasses in the real-world environment. The solutions have proved to benefit the sensation of presence by generating believable behaviours and interaction between the real and virtual objects.

Earlier solutions have developed sophisticated position and orientation tracking systems with position data given an accuracy of less than 1 meter, but in the past, they were often developed for uncomfortable and heavy AR glasses [8]. Smartphone technology comprises multiple components that allow numerous data modalities, enabling the possibility of the same position tracking system made more accessible. Furthermore, the implementation of AR application through industry standard game engines like Unity [Unity3D 2017.30f3], reduces the complexity of development for applications that incorporate such systems and enables the possibility for narrative driven experience completed with real-time visual, haptic, and auditory feedback.

Location-based games can be an experience in which the user and environment are connected through a virtual layer of interactivity. However, games can mediate contextual-awareness, enabling a seamless merge of reality and virtuality. Liminal interface [9] applications can further expand on this experience and create a framing context [10] of which the application takes real life data, merging it with game information so that everyday things can have a new meaning in the context of locationbased gameplay, as represented in Fig. 1. 


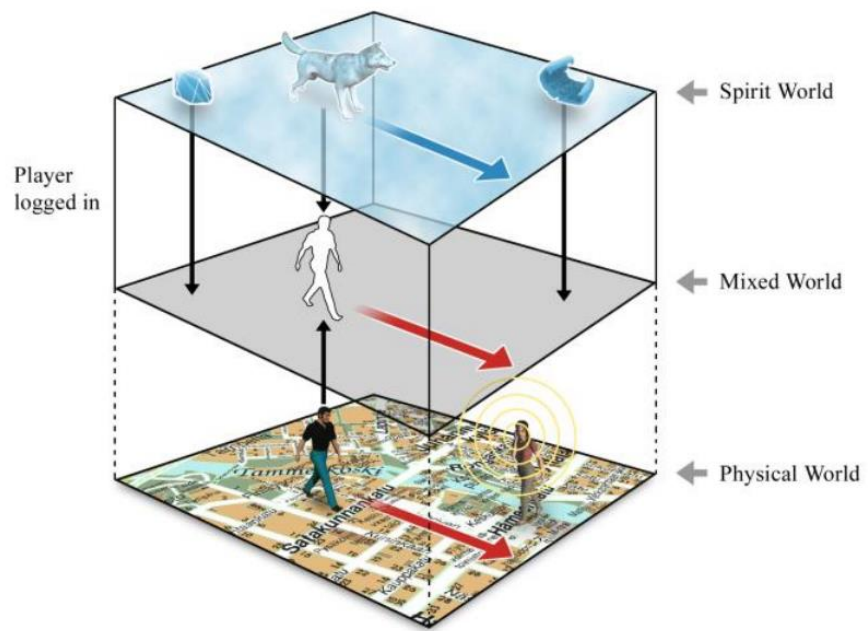

Fig. 1. Frame of Game Reality [11]

This paper focuses on the development of a location-based application that explores an interactive narrative system to help encourage users to be more engaged with an archaeological site. Subsequently, Avebury Portal investigates appropriate methods for delivering the narrative with appropriate use of multimedia and feedback, in the context of an adventurous game with the element of fantasy.

\section{Avebury Portal}

Avebury Portal is a fantasy game developed for the age group of 16-24, intended to be used on-site with a smartphone device and the accompanying 'smart map'. It is designed to be an experiential treasure hunt, guiding users around the site looking for clues that lead them to a 'treasure'. At each point of interest (POI), the user is rewarded with a 'treasure' which contains augmented visual, haptic and/or auditory feedback about their location at time. The narrative is driven by the user and their choice of action (e.g. direction of walking). At each POI during the treasure hunt, the user will need to solve a puzzle or riddle to acquire the 'treasure'. The puzzles creatively use the environment to give clues to the user, and often may quiz the user about a previous encounter instead, to ensure the user is engaged.

Avebury Portal uses Unity engine (Unity3D 2017) and the integrated image-based AR recognition technology (Vuforia [Vuforia]). The engine provides the appropriate toolset for a narrative driven application compatible on Android and iOS mobile operating systems. The prototype uses GPS to track user's location and device compass for orientation, incorporated to generate contextual-awareness throughout the user's treasure hunt. The application is designed to be a prototype, showcasing the proof of concept.

Avebury Portal consists of several parts: The Virtual Narrative, Treasure Hunt and the Multimodal Interface. In 3.1, The Virtual Narrative describes the method 
approached to plan the system's location-based narrative, and in 3.2 Treasure Hunt, we illustrate the design procedure for puzzles, placement of treasures and what information is being delivered in the form of clues. In 3.3, the Multimodal Interface uncovers how the system manages the fusion of GPS and AR for the framing context.

\subsection{The Virtual Narrative}

Avebury Henge consists of a roughly circular earthwork approximately 1 kilometre in circumference, with a ditch on the inside and a bank on the outside, broken into 4 quadrants by entrances that bridge the banks and ditches. The stone circles inside consisted of one large circle on the inside edge of the ditch and two further inner circles surrounding megalithic structures. Most of these stones are no longer present.

The narrative is thematically inspired by the style of fantasy Neolithic folklores and it explores the spirituality and historical aspects of the Henge. Given that this system is a location-aware narrative, it further investigates these concepts with the corresponding environment and presents the information in small packages called 'clues', with room for the user to have freedom of exploration. However, the system is still required to be easily accessible and safe due to the nature of its interaction. This includes the highlight of key iconic places such as: Southern and northern circle, behind the chapel, the cove, etc. They act as key points throughout the hunt and present themselves as safe places if users get lost.

The POIs have supporting information that act as 'treasures'. The presented 'clues' given at these places are extracted from a handy book [12] found in the site's gift shop, combined with various sources that includes more recent and up-to-date archaeological information [13]. In the system design, we refer to every POI as Node, for narrative planning.

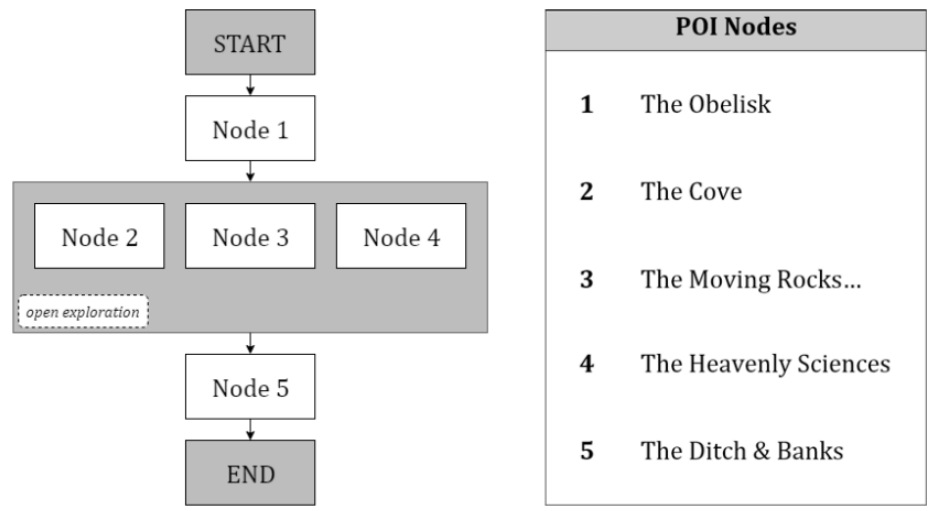

Fig. 2. Treasure Nodes from Avebury Portal

The structure of the narrative can be described as a CDP model and a hybrid of a Canyon-Plain-Canyon [14]. The system allows users to have open exploration in between two independent nodes as shown in Fig. 2. This structure guides users through an environment by controlling their beginning and end but allows immersive freedom of exploration in between [15]. 


\subsection{Treasure Hunt}

The purpose of this hunt is to use AR to enhance engagement with heritage sites of archaeological type. To retain attentiveness of users and interaction between the user and application, we created a map which indicates POIs, known as the 'smart map'. The 'smart map' is provided alongside the application running on a mobile device, encouraging users to find the 'treasures'. The 'treasures' are at POIs placed on the 'smart map', shown as question marks for users to approach. The map contains marker images that are directly linked to each POI for users to scan when they are prompted, demonstrated in Fig.3.

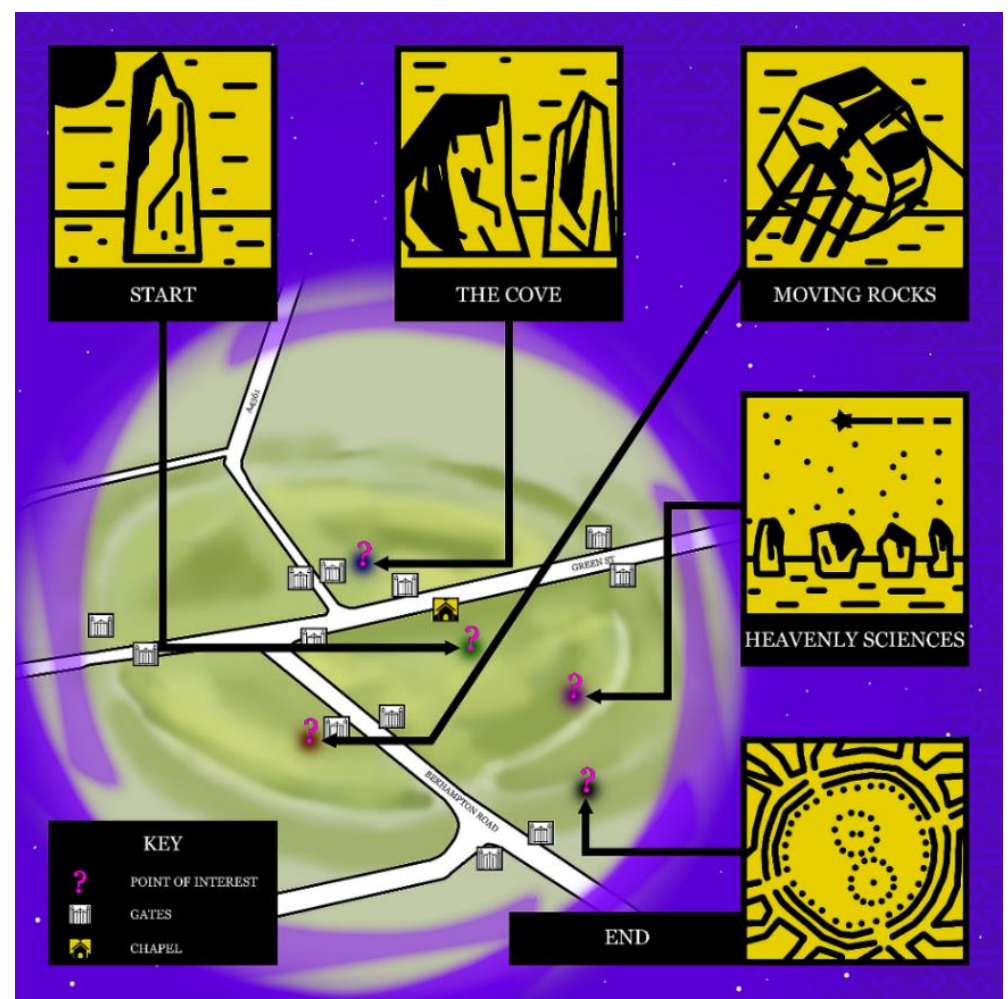

Fig. 3. Smart Map printed to be used with Avebury Portal

At the start of the application, users are asked to approach the chapel to begin the treasure hunt. Avebury Portal demonstrates how the system works by guiding users through the first 'treasure' next to the chapel, which in a way, acts as tutorial. There are 2 clues attached to each 'treasure' found at each POI about Avebury Henge (Fig.5.), illustrated through AR animation, sound and static 3D models. The importance of this system is to ensure users remember the clues they gather from each POI and apply them to solve the puzzle of their sequel POI combined with the directed visual clues found in their environment. However, each user's experience can differ from Node $2-4$ as this is an open exploration section in the narrative structure. The system enables this structure by storing what POI(s) have been visited using an array of Boolean variables 
to identify which puzzle to prompt based on the clues they have gathered from previous POI(s). This is illustrated by an example scenario in Fig. 4.

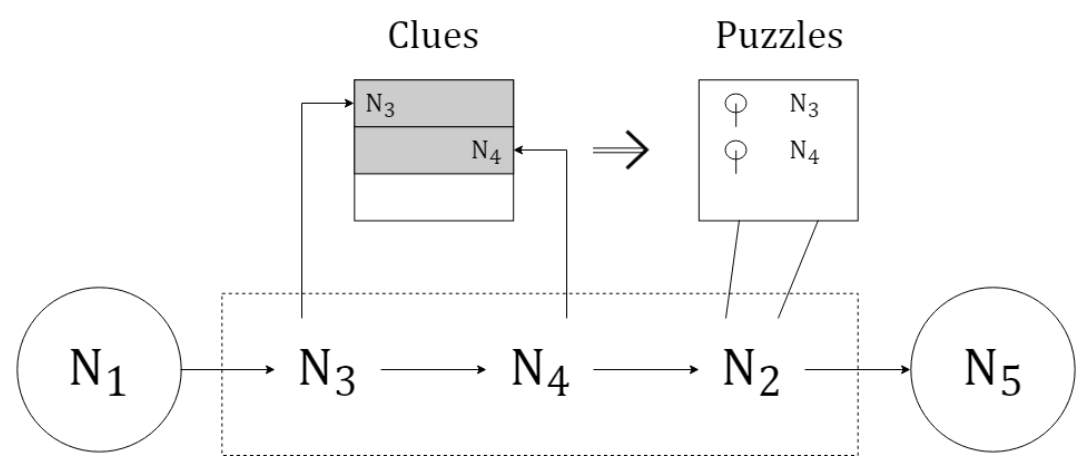

Fig. 4. Example of how clues are collected to be used for the sequel Node.

\begin{tabular}{|cl|}
\hline Node (POI) & \multicolumn{1}{c|}{ Attached Clues } \\
\hline 1 & (a) Age of Avebury (c) Obelisk height \\
2 & (d) The north circle (e) The cove \\
3 & (f) South entrance (g) Moving rocks \\
4 & (h) Cosmic (i) Heavenly numbers \\
5 & (j) The ditches (k) Protecting spirits \\
\hline
\end{tabular}

Fig. 5. Clues attached to each POI

\subsection{Multimodal Interface}

Avebury Portal comprises multimodal inputs to contextualise user interaction with the treasure hunt. An example modality is user's position data, measured by the system and discretised as coordinates to locate user's position at a given instance. Avebury Portal takes the position data of the user and calculates the distance to the pre-set POI coordinates. An area of $10 \mathrm{~m} 2$ is created surrounding each POI, except for Node 5 where it's an area of $5 \mathrm{~m} 2$ (for safety precautions). These areas are made to notify the user when they approach the POI. It calculates the distance between two points where $\varphi$ is latitude, $\lambda$ is longitude, $R$ is earth's radius (mean radius $=6,371 \mathrm{~km}$ ).

$$
\begin{gathered}
\mathrm{a}=\sin ^{2}\left(\frac{\Delta \varphi}{2}\right)+\cos \varphi 1 \cdot \cos \varphi 2 \cdot \sin ^{2}\left(\frac{\Delta \lambda}{2}\right) \\
\mathrm{c}=2 \cdot \operatorname{atan} 2(\sqrt{\mathrm{a}}, \sqrt{(1-\mathrm{a})}) \\
\text { distance }=\mathrm{R} \cdot \mathrm{c}
\end{gathered}
$$




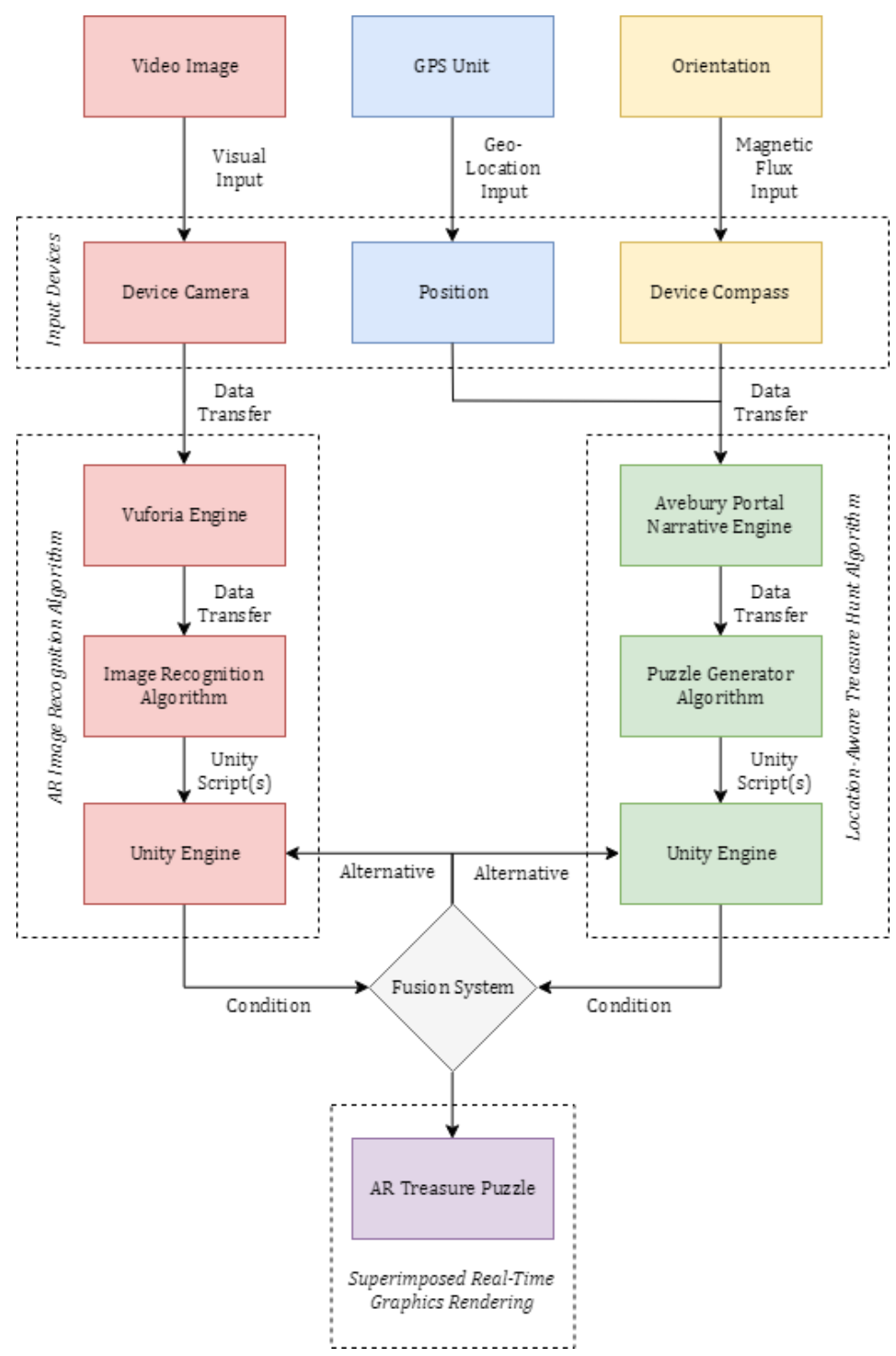

Fig. 6. The fusion scheme for orientation, location, image sensors and algorithms.

Multimodal interface is a method to create an unambiguous format for user interaction by increasing recognition accuracy with data from various modalities, verifying user intention [16]. Multimodal system integration is also referred to as the fusion engine [17]. This idea is expanded further by understanding that modalities with different characteristics may not have obvious ways to connect. Additionally, in [17] Turk concluded, perhaps, the biggest challenge could be the temporal dimension. Some modalities provide information at sparse, discrete points in time (counted) while others generate continuous but less time-specific output (measured). 
In Avebury Portal, the use of this method works to fuse the location-aware narrative engine and puzzle generator algorithm with Vuforia [Vuforia] AR image recognition engine, to output the augmented 'treasures' for contextualised learning experience. Fig.6. demonstrates the fusion scheme for the Location-Aware Treasure Hunt Algorithm and AR Image Recognition Algorithm.

\subsection{Results}

Avebury Portal was tested on-site with 18 participants. Each participant was given up to an hour to complete the treasure hunt whilst they were observed, with notice that they can quit the hunt at any time. The participants were then interviewed and asked to complete a survey. The observations and interviews were recorded to be analysed alongside the survey using the triangulation methodology [18] for further analysis.

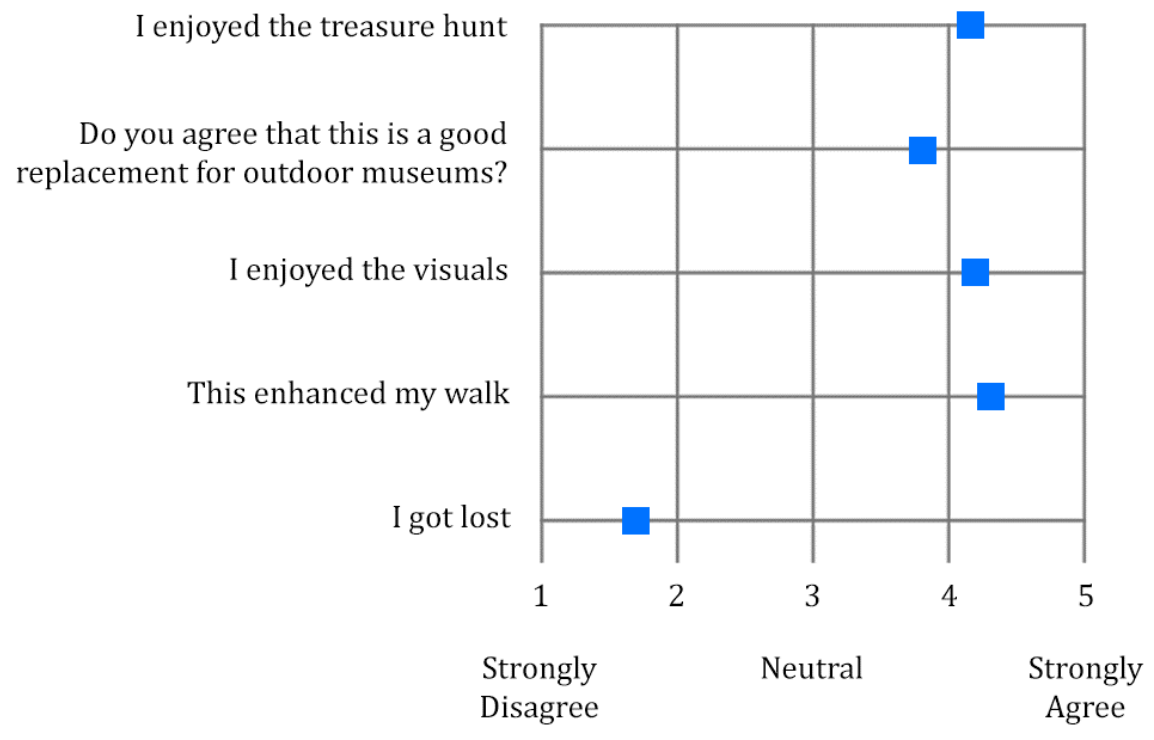

Fig. 7. Mean average of user response for Avebury Portal

The survey results that $50 \%$ of users had experienced a location-based AR game, and that 12 of 18 participants had visited Avebury before. 5 participants were also archaeologist who work on-site. The results of the survey also demonstrate the average response for each statement in Fig.7. Users responded positively to each statement, validated by their actions when observed and backed up by their response in interviews. However, many who responded 1 (Strongly Disagree) to "I got lost" showed some signs of confusion and frustration when they were observed. Perhaps users felt disorientated and confused about their location, suggesting that, they could have been lost. 


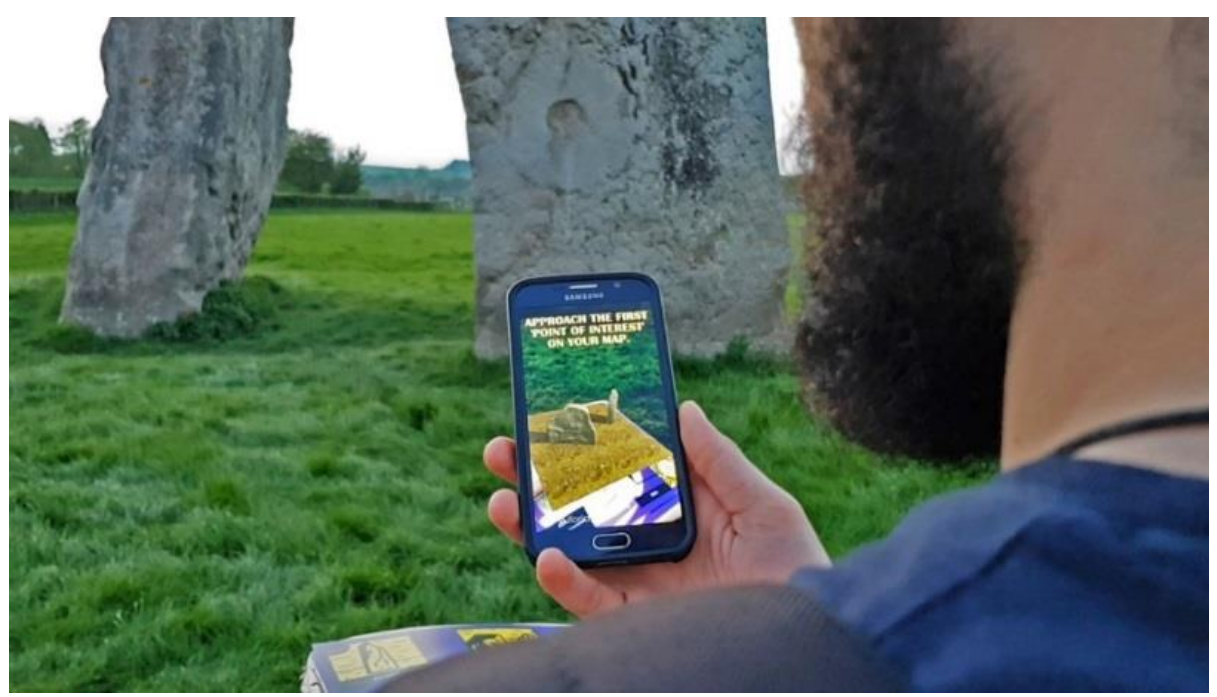

Fig. 8. User interacting with Avebury Portal

\section{Conclusion and Future Work}

In this paper, we manage to design, implement and test a working prototype of a location-based AR treasure hunt that achieves some positive impact on user's experience during their visit to Avebury Henge. Users praised the augmented visuals, agreed that this encouraged their walk at the site, and most said that they feel this could replace current methods for exhibiting an outdoor heritage site. Some users also mentioned that the haptic feedback for notifying users approaching an area of interest was intuitive and appealing. The information given at each clue was clear to the users and they felt that the animations and the riddles were engaging and informative.

However, some users showed signs of confusion when using the map to locate their position, suggesting that perhaps an alternative would be to completely eradicate the 'smart map' and implement in-app navigation system for further guidance of users' location. The system also needs further work on establishing stable and reliable methods for markerless 3D tracking, which could be integrating together with the location-aware system to create a more seamless virtual dimension for users to interact with.

\section{References}

1. UK Department for Digital, Culture Media \& Sport, 2017. Taking Part focus on: Heritage [online]. London: National Statistics. Available from:

https://www.gov.uk/government/uploads/system/uploads/attachment_data/file/655949/Tak ing_Part_Focus_on_Heritage.pdf [Accessed 13 January 2018] 
2. Bay, H., Fasel, B., Gool, LV., 2006. Interactive Museum Guide: Fast and Robust Recognition of Museum Objects [online] Zurich. Computer Vision Laboratory, Zurich. Available from: https://www.vision.ee.ethz.ch/publications/papers/proceedings/eth_biwi_00394.pdf [Accessed 19 February 2017]

3. Niantic., Nintendo, 2016.

4. Chen, P., Liu, X., Cheng, W., Huang, R., 2017. A review of using Augmented Reality in Education from 2011 to 2016 [online]. Beijing, China. Smart Learning Institute, Beijing Normal University, Beijing, China. Available from: https://pdfs.semanticscholar.org/deaa/5b1ad5a3ecec6a83783e7ac371b2a3970ed3.pdf [23 October 2017]

5. Narciso, D., Padua, L., Adao, T., Peres, E., Megalhaes, L., 2015. MixAR Mobile Prototype: Visualizing Virtually Reconstructed Ancient Structures In Situ. Proc. Conference on ENTERprise Information Systems / International Conference on Project MANagement / Conference on Health and Social Care Information Systems and Technologies, CENTERIS / ProjMAN / HCist 2015 October 7-9.

6. English Heritage, 2002. History of Avebury Henge and Stone Circles [online]. Available from: http://www.english-heritage.org.uk/visit/places/avebury/history/ [Accessed 15 March 2017]

7. Papagiannakis, G., Magnenat-Thalmann, N., 2006. Virtual Worlds and Augmented Reality in Cultural Heritage Applications. In: eds. Baltsavias, et al. Proc. Recording, Modeling and Visualization of Cultural Heritage, Abington January 2006. Abington: Taylor and Francis Group. 419-430. Available from: https://www.researchgate.net/publication/232613064_Virtual_Worlds_and_Augmented_R eality_in_Cultural_Heritage_Applications [Accessed 17 Januray 2018].

8. Vlahakis, V., Ioannidis, M., Karigiannis, J., Tsotros, M., Gounaries, M., Stricker, D., Gleue, T., Daehne, P., Almeida, L., 2002. Archeoguide: An Augmented Reality Guide for Archaeological Sites. IEEE 2002. IEEE Computer Graphics and Applications. Volume 22, Issue 5, Sep/Oct 2002, pg. $52-60$.

9. Nieuwdorp, E., 2005. The Pervasive interface: Tracing the Magic Circle. Proc. Digital Games Research Conference 2005, Changing Views: Worlds in Play, Vancouver, Canada 16-20 June 2005.

10. Goffman, E., 1974. Frame Analysis. An Essay on the Organization of Experiences. Boston, USA: Northeastern University Press.

11. Mäyrä, F. and Lankoski, P., 2009. Player in Hybrid Reality. Alternative Approaches in Game Design. In: de Souza e Silva, A., ed. Digital Cityscapes: Merging Digital and Urban Playspaces. 1st edition edition.: Peter Lang Publishing, 129-146

12. Francis, E., 2000. Avebury. Wooden Gift Books.

13. Gillings, M., Barker, D., Pollard, J., Strutt, K., Taylor, J. 2017. Squaring the Circle? Geophysical Survey across part of the Southern Inner Circle of the Avebury.

14. Millard, D.E., Hargood, C., Jewell, M.O., Weal, M.J., 2013. Canyons, deltas and plains: Towards a unified sculptural model of location-based hypertext. In: Proceedings of the 24th ACM Conference on Hypertext and Social Media.

15. Hargood, C., Hunt, V., Weal, M., Millard, D.E., 2016. Patterns of sculptural hypertext in location based narratives. In: Proceedings of the 27th ACM Conference on Hypertext and Social Media. ACM, New York, NY, USA. 
16. Kurschl, W., Gottesheim, W., Mitsch, S., Prokop, R., Schönböck, J., 2007. Proposing Usability Patterns for Mobile Multimodal Applications. Hagenberg, Austria, 2007.

17. Turk, M., 2013. Pattern Recognition Letters. Multimodal interaction: A review [online], In Press. Available from: http://www.cs.ucsb.edu/ mturk/Papers/TurkPRL2013.pdf [Accessed 12 November 2017].

18. Hsieh, MC., Lin, HC., 2011. A Conceptual Study for Augmented Reality E-learning System based on Usability Evaluation. In. Communications in Information Science and Management Engineering. 